\title{
GROUND-BASED MULTISITE OBSERVATIONS OF TWO TRANSITS OF HD 80606b
}

\author{
A. Shporer ${ }^{1,2}$, J. N. Winn ${ }^{3}$, S. Dreizler ${ }^{4}$, K. D. Colón 5 , W. M. Wood-Vasey ${ }^{6}$, P. I. Choi ${ }^{7}$, C. Morley ${ }^{8}$, C. Moutou Mou $^{9}$, \\ W. F. Welsh ${ }^{10}$, D. Pollaco ${ }^{11}$, D. Starkey ${ }^{12}$, E. Adams ${ }^{8}$, S. C. C. Barros ${ }^{11}$, F. Bouchy ${ }^{13,14}$, A. Cabrera-Lavers ${ }^{15,16}$, \\ S. Cerutti ${ }^{6}$, L. Coban ${ }^{6}$, K. Costello $^{6}$, H. Deeg ${ }^{15}, 17$, R. F. Díaz ${ }^{13}$, G. A. Esquerdo ${ }^{18}$, J. Fernandez ${ }^{4}$, S. W. Fleming ${ }^{5}$,

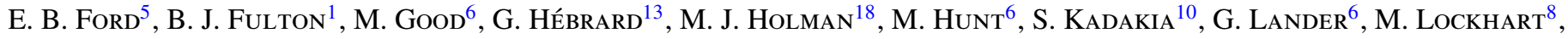 \\ T. Mazeh ${ }^{19}$, R. C. Morehead ${ }^{5}$, B. E. Nelson ${ }^{5}$, L. Nortmann ${ }^{4}$, F. Reyes ${ }^{5}$, E. RoebucK ${ }^{6}$, A. R. Rudy ${ }^{7}$, R. Ruth ${ }^{5}$, E. Simpson ${ }^{11}$, \\ C. VINCENT ${ }^{6}$, G. WEAVER ${ }^{6}$, AND J.-W. XIE ${ }^{5}$ \\ ${ }^{1}$ Las Cumbres Observatory Global Telescope Network, 6740 Cortona Drive, Suite 102, Santa Barbara, CA 93117, USA; ashporer@1cogt.net \\ ${ }^{2}$ Department of Physics, Broida Hall, University of California, Santa Barbara, CA 93106, USA \\ ${ }^{3}$ Department of Physics and Kavli Institute for Astrophysics and Space Research, Massachusetts Institute of Technology, Cambridge, MA 02139, USA \\ ${ }^{4}$ Georg-August-Universität, Institut für Astrophysik, Friedrich-Hund-Platz 1, 37077 Göttingen, Germany \\ ${ }^{5}$ Department of Astronomy, University of Florida, 211 Bryant Space Science Center, Gainesville, FL 32611-2055, USA \\ ${ }^{6}$ Department of Physics and Astronomy, University of Pittsburgh, 3941 O'Hara Street, Pittsburgh, PA 15260, USA \\ ${ }^{7}$ Department of Physics and Astronomy, Pomona College, 610 North College Avenue, Claremont, CA 91711, USA \\ ${ }^{8}$ Department of Earth, Atmospheric, and Planetary Sciences, Massachusetts Institute of Technology, 77 Massachusetts Avenue, Cambridge, MA 02139, USA \\ ${ }^{9}$ Laboratoire d'Astrophysique de Marseille, Université de Provence, CNRS (UMR 6110), 38 rue Frédéric Joliot Curie, 13388 Marseille Cedex 13, France \\ ${ }^{10}$ Department of Astronomy, San Diego State University, 5500 Campanile Drive, San Diego, CA 92182, USA \\ ${ }^{11}$ Astrophysics Research Centre, School of Mathematics \& Physics, Queens University, University Road, Belfast, BT7 1NN, UK \\ 12 DeKalb Observatory H63, Auburn, IN 46706, USA \\ ${ }^{13}$ Institut d'Astrophysique de Paris, UMR7095 CNRS, Université Pierre \& Marie Curie, 98 bis boulevard Arago, 75014 Paris, France \\ ${ }^{14}$ Observatoire de Haute-Provence, CNRS/OAMP, 04870 Saint-Michel-l'Observatoire, France \\ ${ }^{15}$ Instituto de Astrofísica de Canarias, C. Via Lactea S/N, 38205 La Laguna, Tenerife, Spain \\ ${ }^{16}$ GTC Project Office, E-38205 La Laguna, Tenerife, Spain \\ ${ }^{17}$ Departamento de Astrofísica, Universidad de La Laguna, 38200 La Laguna, Tenerife, Spain \\ ${ }^{18}$ Harvard-Smithsonian Center for Astrophysics, Cambridge, MA 02138, USA \\ ${ }^{19}$ Wise Observatory, Tel Aviv University, Tel Aviv 69978, Israel \\ Received 2010 July 19; accepted 2010 August 19; published 2010 September 23
}

\begin{abstract}
We present ground-based optical observations of the 2009 September and 2010 January transits of HD 80606b. Based on three partial light curves of the 2009 September event, we derive a midtransit time of $T_{c}$ [HJD] $=$ $2455099.196 \pm 0.026$, which is about $1 \sigma$ away from the previously predicted time. We observed the 2010 January event from nine different locations, with most phases of the transit being observed by at least three different teams. We determine a midtransit time of $T_{c}[\mathrm{HJD}]=2455210.6502 \pm 0.0064$, which is within $1.3 \sigma$ of the time derived from a Spitzer observation of the same event.
\end{abstract}

Key words: planetary systems - stars: individual (HD 80606)

Online-only material: color figure, machine-readable table

\section{INTRODUCTION}

The sample of transiting exoplanets has grown rapidly in recent years, but HD 80606b stands out from this crowd by virtue of its long period (111.4 days) and high orbital eccentricity $(e=0.93)$. Initially, the planet was discovered in a Doppler survey and was not known to transit (Naef et al. 2001). However, the planet is near pericenter during superior conjunctions, increasing the probability of occultations (secondary eclipses). This motivated the observations of Laughlin et al. (2009), who found that the orbital inclination is indeed close enough to $90^{\circ}$ for occultations to occur. Soon after, the discovery of a transit (primary eclipse) was reported by several authors (Moutou et al. 2009; Fossey et al. 2009; Garcia-Melendo \& McCullough 2009). In addition, the orbit was shown to be misaligned with the plane defined by the stellar rotation (Moutou et al. 2009; Pont et al. 2009; Winn et al. 2009; Hébrard et al. 2010). This system has become an important case study for theories of orbital migration, tidal interactions, and giant planet atmospheres (e.g., Wu \& Murray 2003; Fabrycky \& Tremaine 2007; Triaud et al. 2010; Pont et al. 2008; Laughlin et al. 2009; Knutson et al. 2009).
For any transiting planet, it is desirable to observe multiple transits. This allows the system parameters to be refined, especially the orbital period. In addition, a deviation from strict periodicity may be the signature of additional bodies in the system, such as another planet or a satellite (e.g., Holman \& Murray 2005; Agol et al. 2005; Simon et al. 2007; Nesvorný \& Beaugé 2010).

In the case of HD 80606b observations of complete transits are challenging. This is partly because transits are rare, occurring once every 111.4 days, and also because the transit duration is nearly $12 \mathrm{hr}$, making it impossible to be observed in entirety from a single ground-based site. Hidas et al. (2010) and Winn et al. (2009) carried out multisite campaigns to achieve more complete coverage of the transit. This paper reports the results of our more recent observations. In 2009 September, only the first portion of the transit could be observed, but our combined light curve for 2010 January ranges over the entire transit, with most phases having been observed with at least three different telescopes.

Hébrard et al. (2010) utilized the Spitzer observatory to obtain a high-quality light curve of the entire 2010 January transit event. Besides deriving refined system parameters, they measured a 
midtransit time earlier by $3 \sigma$ than was predicted by Winn et al. (2009). This discrepancy suggested the intriguing possibility of the existence of a third body in the HD 80606 transiting system. The primary goal of our analysis was to determine the times of these events as accurately as possible. Although our results are not as precise as the Spitzer result, we provide an independent estimate of the midtransit time. Our observations and photometric processing are described in Section 2. In Section 3, we describe our analysis, the results of which are presented in Section 4 and discussed in Section 5.

\section{OBSERVATIONS}

The new data presented in this paper are three photometric time series spanning the first portion of the transit of 2009 September 23/24 (hereafter Sep09), based on observations across North America; and ten photometric time series spanning the entire transit of 2010 January 13/14 (hereafter Jan10), based on data from Israel, Europe, Canary Islands, North America, and Hawaii.

In all cases, we gathered CCD images encompassing the target star, HD 80606 (G5V, $V=9.06, B-V=0.76$ ), and its visual binary companion, HD 80607 (G5V, $V=9.07, B-V=$ 0.87 ), which is located $21^{\prime \prime}$ east. The similarity in brightness and color between the two stars facilitates differential photometry. Since the stars are relatively bright, we defocused most of the telescopes that were used, thereby allowing for longer exposures without saturation and reducing the impact of pixel sensitivity variations and seeing variations. We always ensured that the point-spread functions (PSFs) of the two stars were well separated. Brief descriptions of the observations from each site are given below, along with an abbreviated observatory name that we will use in the remainder of this paper.

Wise Observatory ${ }^{20}$ (WO), Israel. The target was observed with the $1.0 \mathrm{~m}$ telescope for $9 \mathrm{hr}$ on the Jan10 transit night, completely covering ingress, and for $2 \mathrm{hr}$ on the following night. An RGO $Z$ filter was used and the telescope was slightly defocused. The detector was a back-illuminated Princeton Instruments CCD, with a $12.6 \times 13$. 0 field of view $(\mathrm{FOV})$ and a pixel scale of 0. . $^{\prime} 580$ pixel $^{-1}$.

Gran Telescopio Canarias ${ }^{21}$ (GTC), La Palma, Canary Islands. We observed the target with the Optical System for Imaging and low Resolution Integrated Spectroscopy (OSIRIS), mounted on the $10.4 \mathrm{~m} \mathrm{GTC}$ telescope. The observations lasted for $8.3 \mathrm{hr}$ on the Jan 10 transit night, all within the transit. A small defocus was applied, resulting in a typical PSF FWHM of $1^{\prime \prime} .5$. We used the narrowband imager with the red range tunable filter, alternating between four narrow bands in the range $7680-7780 \AA$, each with an FWHM of $12 \AA$. The instrument has a maximum FOV of 7! $8 \times 7.8$ and a pixel scale of $0 .{ }^{\prime \prime} 127$ pixel $^{-1}$. Analysis of the light curve for each individual narrowband filter is presented in Colón et al. (2010); here, we are concerned with the light curve based on a summation of the flux observed in all four filters. We adopt the SDSS- $i^{\prime}$ band limb-darkening coefficients when fitting this light curve.

Observatoire de Haute Provence ${ }^{22}(O H P)$, France. The target was observed with the $1.2 \mathrm{~m}$ telescope for $4.3 \mathrm{hr}$ during the first half of the Jan10 transit, with no out-of-transit observations. As was the case with the spectroscopic observations secured simultaneously with Sophie at OHP (Hébrard et al. 2010),

\footnotetext{
20 http://wise-obs.tau.ac.il/

21 http://www.gtc.iac.es/en/pages/gtc.php

22 http://www.obs-hp.fr
}

the transit sequence had to be stopped due to cloudy weather. Photometric observations were also secured on several nights shortly before and after the transit night to put constraints on the stellar activity. These data are described in more detail by Hébrard et al. (2010). No defocus was applied here; instead, to allow for longer exposures, we used a neutral density filter along with a Gunn- $r$ filter.

Allegheny Observatory ${ }^{23}$ (AO), Pittsburgh, PA, USA. We used the $0.41 \mathrm{~m}$ (16 inch) Meade telescope and an SBIG 0." 56 pixel $^{-1}$ CCD, with an FOV of $20{ }^{\prime} 0 \times 30{ }^{\prime} 0$. Observations of the Jan 10 transit were done in the $I$ filter with no defocus, from the time of second contact until about $2 \mathrm{hr}$ after the transit ended, a total of $11.4 \mathrm{hr}$.

Rosemary Hill Observatory ${ }^{24}$ (RHO), Bronson, FL, USA. We used the $0.76 \mathrm{~m}$ Tinsley telescope and an SBIG ST-402ME CCD camera mounted at the $f / 4$ Newtonian focus, with an FOV of $3.88 \times 2.56$ (half of the entire FOV). During the Jan10 event, we observed for $4.9 \mathrm{hr}$ with no out-of-transit data. We used the same instrument to observe the Sep09 event, obtaining $2.4 \mathrm{hr}$ of data just before the transit started, which helps constrain the transit start time. Both events were observed with the SDSS $-i^{\prime}$ filter and the telescope was defocused.

Fred Lawrence Whipple Observatory ${ }^{25}$ (FLWO), Mt. Hopkins, AZ, USA. We used Keplercam, mounted on the $1.2 \mathrm{~m}$ telescope, and observed the target for $2.8 \mathrm{hr}$ during the flat bottom part of the Jan10 transit. The same instrument was also used to observe the very beginning of the Sep09 event, obtaining $1.8 \mathrm{hr}$ of data. Keplercam includes a $4 \mathrm{~K} \times 4 \mathrm{~K}$ Fairchild

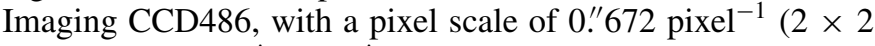
binning) and a 23! $1 \times 23 ! 1$ FOV. FLWO observations of both transit events used the SDSS $-i^{\prime}$ filter and started immediately after the target rose.

Table Mountain Observatory ${ }^{26}$ (TMO), Wrightwood, CA, $U S A$. We used the $1.0 \mathrm{~m}$ telescope with an Apogee U16 4K $\times 4 \mathrm{~K}$ CCD, with a pixel scale of $0 . \prime 18$ pixel $^{-1}$ and a $12 ! 3 \times 12 ! 3$ FOV. The SDSS $-i^{\prime}$ filter was used here. TMO observations completely cover the Jan 10 egress, with a total of $6.6 \mathrm{hr}$, of which $2.9 \mathrm{hr}$ are after fourth contact.

George R. Wallace Jr. Astrophysical Observatory ${ }^{27}$ (WAO), Westford, MA, USA. We used two identical $0.36 \mathrm{~m}$ telescopes on the Jan10 transit night, each equipped with an SBIG STL$1001 \mathrm{E} 1 \mathrm{~K} \times 1 \mathrm{~K} \mathrm{CCD}$ camera with a pixel scale of $1^{\prime \prime} .29 \mathrm{pixel}^{-1}$, resulting in an FOV of $21.5 \times 21.5$. We refer to these instruments as WAO-1 and WAO-2. At both telescopes, we observed in the $I$ filter with no defocus. With WAO-1 we were able to observe for 15 minutes during the flat bottom part, before observing conditions degraded. Observations resumed with both instruments at the time of third contact and continued for $4.2 \mathrm{hr}$, of which $2.4 \mathrm{hr}$ were out-of-transit.

Faulkes Telescope North ${ }^{28}$ (FTN), Mt. Haleakala, Maui, HI, USA. We used the LCOGT ${ }^{29} 2.0 \mathrm{~m}$ FTN telescope and the Spectral Instruments camera with the Pan-STARRS $Z$ filter. The camera has a back-illuminated Fairchild Imaging CCD, and we used the default $2 \times 2$ pixel binning mode, with an effective pixel scale of $0 . " 304$ pixel $^{-1}$. The telescope was defocused and the $10.5 \times 10$ '. 5 FOV was positioned and rotated so that the guiding

\footnotetext{
23 http://www.pitt.edu/ aobsvtry/

24 http://www.astro.ufl.edu/information/rho.html

25 http://www.sao.arizona.edu/FLWO/whipple.html

26 http://tmoa.jpl.nasa.gov/

27 http://web.mit.edu/wallace/

28 http://faulkes-telescope.com/

29 http://lcogt.net
} 
Table 1

HD 80606 Light Curves Presented in This Work

\begin{tabular}{cccc}
\hline \hline HJD & \multicolumn{2}{c}{ Flux Ratio } & \multicolumn{2}{c}{ Error } & Observatory $^{\mathrm{a}}$ \\
\hline 2455210.288327 & 1.00114 & 0.00116 & \\
2455210.445392 & 1.11691 & 0.00024 & WO \\
2455210.446272 & 0.99860 & 0.00106 & GTC \\
2455210.515999 & 1.10749 & 0.00406 & OHP \\
2455210.607419 & 1.11228 & 0.00107 & AO \\
2455210.654098 & 1.11259 & 0.00110 & RHO \\
2455210.748739 & 1.11077 & 0.00142 & FLWO \\
2455210.588711 & 1.10630 & 0.00116 & TMO \\
2455210.815045 & 1.11449 & 0.00134 & WAO-1 \\
2455209.863305 & 0.99977 & 0.00037 & WAO-2 \\
\hline & 2009 Sep transit event & FTN \\
\hline 2455098.867697 & 1.12197 & 0.00227 & RHO \\
2455098.950735 & 1.11411 & 0.00138 & FLWO \\
2455098.974510 & 1.12694 & 0.00101 & MLO \\
\hline
\end{tabular}

Note. ${ }^{a}$ Observatories name abbreviations: WO: Wise Observatory; GTC: Grand Telescopio Canarias; OHP: Observatoire de Haute Provence; AO: Allegheny Observatory; RHO: Rosemary Hill Observatory; FLWO: Fred Lawrence Whipple Observatory; TMO: Table Mountain Observatory; WAO: Wallace Astrophysical Observatory; FTN: Faulkes Telescope North; MLO: Mountain Laguna Observatory.

(This table is available in its entirety in a machine-readable form in the online journal. A portion is shown here for guidance regarding its form and content.)

camera FOV will contain a suitable guide star. We observed the target on the Jan 10 transit night for $9.2 \mathrm{hr}$, from the beginning of egress until $7 \mathrm{hr}$ after the transit ended. We also observed during the two adjacent nights: for $2 \mathrm{hr}$ on the preceding night (January $12 / 13$ ) and for $3.5 \mathrm{hr}$ on the following night (January 14/15).

Mount Laguna Observatory ${ }^{30}$ (MLO), San Diego, CA, USA. Only the Sep09 event was observed. We used the $1.0 \mathrm{~m}$ telescope with a Fairchild Imaging $2 \mathrm{~K} \times 2 \mathrm{~K}$ CCD and the SDSS $-i^{\prime}$ filter. Observations were done with a $300 \times 300$ pixel subarray and an FOV of $2.0 \times 2 ! 0$. The telescope was defocused and the very beginning of the event was observed, for $1.6 \mathrm{hr}$ with no out-of-transit data.

Four additional light curves were obtained for the Jan 10 event by the Liverpool Telescope, MONET-North telescope, DeKalb Observatory, and a third telescope at the WAO, and one for the Sep09 event by the AO. However, those data displayed a very high noise level and strong systematic effects and were not included in our subsequent analysis.

The CCD data were reduced using standard routines for bias subtraction, dark current subtraction (when necessary), and flatfield correction. We used aperture photometry to derive the flux of HD 80606 and HD 80607 and divided the former by the latter to obtain a time history of the flux ratio, which we refer to as the light curve. We took care to choose aperture sizes to avoid contamination of one stellar signal by the other star. Our time stamps represent the Heliocentric Julian Date, based on the UTC at midexposure (and not the uniformly flowing terrestrial time system advocated by Eastman et al. 2010). This is also the time system that was used for the Spitzer analysis of Hébrard et al. (2010).

All light curves were averaged into 10 minute bins, using $3 \sigma$ outlier rejection. The error bar assigned to each data point

\footnotetext{
30 http://mintaka.sdsu.edu/
}

was the standard deviation of the mean of all the measurements contributing to each 10 minute bin, which ranged in number from 4 to 63 . There is no significant information loss due to time binning, because the binning time of 10 minutes is shorter than the duration of ingress and egress by more than an order of magnitude.

The data only cover the first portion of the 2009 September $23 / 24$ event, but they provide a complete coverage of the 2010 January $13 / 14$ event. Although the first hour of the transit was observed from only one observatory (WO), the rest of the transit was observed by $3-5$ different sites, which is very helpful for identifying and decreasing the influence of any systematic effects that are specific to each observatory (i.e., correlated noise or red noise) which is frequently a problem with ground-based photometric data (e.g., Pont et al. 2006; Carter \& Winn 2009; Sybilski et al. 2010).

Table 1 gives the photometric data that were obtained and analyzed. Each data point represents a 10 minute binned average of the flux ratio of HD 80606 to HD 80607. The normalization factors and error rescaling factors that are described in Section 3 were not applied to the data given in the table. Table 2 gives a list of all the observatories.

\section{DATA ANALYSIS}

In this section, we describe the methods by which we combined the data and derived the midtransit time of each event. We describe the process in detail for the Jan10 event; the details were very similar for the Sep09 data.

Because the quality of the Spitzer light curve is superior to any ground-based light curve, we did not attempt to use our data to refine the basic system parameters other than the midtransit times. Instead, we used the parameters derived by Hébrard et al. (2010) as constraints on the light-curve shape, while allowing the midtransit time to be a free parameter, as described below.

A simultaneous analysis of several light curves obtained by different instruments is a challenging task. Winn et al. (2009) and Hidas et al. (2010) have carried out a similar task, although for a smaller number of datasets. One of the crucial points is the placement of the different flux ratio light curves onto the same scale, despite the differences in bandpasses, detectors, and weather conditions at each observatory. One way of thinking about this problem is that we need to establish the out-of-transit flux ratio that was measured, or that would be measured, by each observatory. Winn et al. (2009) performed this calibration by using data taken on nights when the planet was not transiting, a method that may be affected by night-tonight variations due to varying observing conditions. Hidas et al. (2010) allowed the overall flux ratio scale to be a free parameter for each light curve, thereby increasing the overall number of fitted parameters. Here we chose an intermediate approach. We assigned a normalization factor to each light curve, estimating it from the out-of-transit data whenever possible and allowing it to be a free parameter when there was insufficient out-of-transit data.

We divided our light curves into three groups, based on the amount of out-of-transit data.

Group I includes the light curves with abundant out-of-transit information. The only members of this group are the WO and FTN light curves of the Jan10 event, where out-of-transit measurements were obtained on the transit night and on one (for WO) or two (for FTN) of the adjacent nights. For each of the two light curves separately, we subsequently fitted a seconddegree polynomial to the out-of-transit flux ratio points versus 
Table 2

Observatories List

\begin{tabular}{|c|c|c|c|c|c|c|c|c|c|c|c|c|}
\hline No. & Obs. & $\begin{array}{l}\text { Tel. } \\
(\mathrm{m})\end{array}$ & Filter & \multicolumn{5}{|c|}{$\begin{array}{c}\text { Transit Part }^{\mathrm{a}} \\
\text { OIBEO }\end{array}$} & $\alpha$ & $\bar{\beta}$ & $\begin{array}{c}\mathrm{RMS}^{\mathrm{b}} \\
(\%)\end{array}$ & $\begin{array}{c}\mathrm{PNR}^{\mathrm{c}} \\
\left(\% \text { minute }^{-1}\right)\end{array}$ \\
\hline \multicolumn{13}{|c|}{$2010 \mathrm{Jan}$} \\
\hline 1 & WO & 1.0 & $Z$ & $\mathrm{O}$ & I & $\mathrm{B}$ & $\ldots$ & $\ldots$ & 1.45 & 1.00 & 0.11 & 0.29 \\
\hline 2 & GTC & 10.4 & $7680-7780 \AA\left(i^{\prime}\right)$ & $\ldots$ & I & $\mathrm{B}$ & $\mathrm{E}$ & $\ldots$ & 3.29 & 2.15 & 0.05 & 0.09 \\
\hline 3 & OHP & 1.2 & Gunn- $r+\mathrm{ND}^{\mathrm{d}}$ & $\ldots$ & I & B & $\ldots$ & $\ldots$ & 2.49 & 2.27 & 0.13 & 0.25 \\
\hline 4 & $\mathrm{AO}$ & 0.41 & $I$ & $\ldots$ & $\ldots$ & B & $\mathrm{E}$ & $\mathrm{O}$ & 1.00 & 1.10 & 0.12 & 0.43 \\
\hline 5 & RHO & 0.76 & $i^{\prime}$ & $\ldots$ & $\ldots$ & B & $\mathrm{E}$ & $\ldots$ & 1.91 & 2.59 & 0.09 & 0.15 \\
\hline 6 & FLWO & 1.2 & $i^{\prime}$ & $\ldots$ & $\ldots$ & B & $\ldots$ & $\ldots$ & 1.11 & 1.53 & 0.08 & 0.21 \\
\hline 7 & TMO & 1.0 & $i^{\prime}$ & $\ldots$ & $\ldots$ & B & $\mathrm{E}$ & $\mathrm{O}$ & 1.04 & 1.18 & 0.07 & 0.20 \\
\hline 8 & WAO-1 & $0.36^{\mathrm{e}}$ & $I$ & $\ldots$ & $\ldots$ & B & $\mathrm{E}$ & $\mathrm{O}$ & 1.64 & 1.00 & 0.16 & 0.33 \\
\hline 9 & WAO-2 & $0.36^{\mathrm{e}}$ & $I$ & $\ldots$ & $\ldots$ & $\ldots$ & $\mathrm{E}$ & $\mathrm{O}$ & 1.25 & 1.35 & 0.11 & 0.28 \\
\hline 10 & FTN & 2.0 & $Z$ & $\ldots$ & $\ldots$ & $\ldots$ & $\mathrm{E}$ & $\mathrm{O}$ & 1.24 & 1.07 & 0.07 & 0.23 \\
\hline \multicolumn{13}{|c|}{2009 Sep } \\
\hline 1 & RHO & 0.76 & $i^{\prime}$ & $\mathrm{O}$ & $\ldots$ & $\ldots$ & $\ldots$ & $\ldots$ & 1.00 & 1.00 & 0.12 & 0.44 \\
\hline 2 & FLWO & 1.2 & $i^{\prime}$ & $\mathrm{O}$ & I & $\ldots$ & $\ldots$ & $\ldots$ & 1.00 & 1.00 & 0.08 & 0.30 \\
\hline 3 & MLO & 1.0 & $i^{\prime}$ & $\mathrm{O}$ & I & $\ldots$ & $\ldots$ & $\ldots$ & 1.06 & 1.00 & 0.09 & 0.23 \\
\hline
\end{tabular}

Notes.

a OIBEO for out-of-transit before ingress, ingress, flat bottom, egress, and out-of-transit after egress.

${ }^{b}$ rms residual of the 10 minute binned light curve.

c Photometric noise rate of the unbinned light curve, calculated as $\mathrm{rms} / \sqrt{\Gamma}$, where $\Gamma$ is the median number of exposures per minute.

d Neutral density filter.

e Two identical telescopes.

air mass, time, and PSF FWHM, and divided the entire light curve by this polynomial. The effect of this process on the intransit points was small, typically at the few $0.01 \%$ level. The assigned normalization factor for each of the two resulting light curves was 1.0.

Group II includes light curves that have at least $1.5 \mathrm{hr}$ of outof-transit measurements. Their normalization factors were taken to be the mean out-of-transit flux ratio. This group includes four light curves: the AO, the TMO, and the two WAO light curves.

Group III includes the remaining four light curves (GTC, OHP, RHO, and FLWO) with either a small amount of data or no out-of-transit data at all. The normalization factors were taken to be free parameters in our model.

Our model for the data is based on the premise of two spherical objects, a non-luminous planet, and a limb-darkened star with a quadratic limb-darkening law, in an eccentric Keplerian orbit. For each binned time stamp, we calculated the sky-projected planet-star distance and used the equations of Mandel \& Agol (2002) to calculate the relative flux at that time. Our code accounts for the light travel time effects described by Hébrard et al. (2010).

The model included a total of 34 parameters: orbital period $P$; planet-to-star radius ratio $r=R_{\mathrm{p}} / R_{\mathrm{s}}$; orbital semimajor axis in units of the stellar radius $a / R_{\mathrm{s}}$; orbital eccentricity $e$; argument of periastron $\omega$ (in fact, our fitting parameters were actually $e \cos \omega$ and $e \sin \omega)$; inclination angle $i$; an individual periastron passage time $\left\{T_{p, i}\right\}_{i=1}^{10}$, for each of the 10 light curves (which were later converted into midtransit times); two limb-darkening coefficients $u_{1}$ and $u_{2}$, for each of the different four filters used; and 10 normalization factors, one for each light curve.

The limb-darkening coefficients were estimated from the grids of Claret $(2000,2004)$ for a star with $T_{\text {eff }}=5645 \mathrm{~K}$, $\log g=4.5$, and $[\mathrm{Fe} / \mathrm{H}]=0.43$ (Naef et al. 2001) and were held fixed in the fitting process as the data are insensitive to the coefficients. As mentioned earlier, we used the parameters of Hébrard et al. (2010) to constrain the light-curve shape. We used their values and uncertainties for $P, r, a / R_{s}, e \cos \omega, e \sin \omega$, and $i$ as a priori Gaussian constraints by adding penalty terms to the $\chi^{2}$-fitting statistics. We constrained in a similar way the normalization factors of the six light curves in Groups I and II described above, while assuming a normalization factor uncertainty of $0.1 \%$. This uncertainty is larger than that of the light curves mean out-of-transit flux ratio, typically a few times $0.01 \%$; and it was used in order to prevent the fitting process from being dominated by a single light curve.

Out of the 34 model parameters, 8 were held fixed (the limbdarkening coefficients), 12 were controlled mainly by Gaussian priors $\left(P, r, a / R_{s}, e \cos \omega, e \sin \omega, i\right.$, and six normalization factors), and the remaining 14 were free parameters with uniform priors $\left(\left\{T_{p, i}\right\}_{i=1}^{10}\right.$ and four normalization factors). Our fitting statistic was

$$
\chi^{2}=\chi_{f}^{2}+\chi_{\mathrm{orb}}^{2}+\chi_{\mathrm{norm}}^{2},
$$

where the first term on the right-hand side is the usual $\chi^{2}$ statistics:

$$
\chi_{f}^{2}=\sum_{i=1}^{431}\left[\frac{f_{i}(\mathrm{obs})-f_{i}(\text { model })}{\sigma_{f_{i}}}\right]^{2},
$$

the second term includes the penalties for the light-curve parameters (values and uncertainties taken from Hébrard et al. 2010, $P$ in days and $i$ in degrees):

$$
\begin{aligned}
\chi_{\text {orb }}^{2}= & {\left[\frac{P-111.4367}{0.0004}\right]^{2}+\left[\frac{r-0.1001}{0.0006}\right]^{2} } \\
& +\left[\frac{a / R_{S}-97.0}{1.6}\right]^{2}+\left[\frac{e \cos \omega-0.4774}{0.0018}\right]^{2} \\
& +\left[\frac{e \sin \omega-(-0.8016)}{0.0017}\right]^{2}+\left[\frac{i-89.269}{0.018}\right]^{2},
\end{aligned}
$$


and the third term constrained the normalization factors of the six light curves for which we have sufficient out-of-transit data:

$$
\chi_{\text {norm }}^{2}=\sum_{l=1}^{6}\left[\frac{f_{\text {oot }, 1}-\bar{f}_{\text {oot }, 1}}{0.001}\right]^{2} .
$$

After a preliminary fit, the residuals of each dataset were carefully examined. Two data points were clear outliers, departing from the model by $>4 \sigma$, and were rejected, leaving a total of 431 points. The rejected points were either the first or last data points in the time series and were probably affected by the high air mass or relatively bright twilight sky. After refitting, there were no additional $>4 \sigma$ outliers.

Next, for the purpose of determining parameter uncertainties, we determined appropriate weights for each data point. This was done in two steps specific to each light curve. First, we rescaled the error bars such that the median error bar was equal to the rms residual. We named this rescaling factor $\alpha$. If the median error bar was already equal to or smaller than the rms residual, we set $\alpha=1.0$. Second, we attempted to account for correlated (red) noise in each time series on the critical timescale of the ingress/ egress duration $(2.8 \mathrm{hr})$. We used the "time-averaging" method (e.g., Winn et al. 2008), in which the residuals are binned using several bin sizes, close to the duration of ingress and egress. The amount of correlated noise is then quantified by the ratio between the binned residual light curves standard deviation and the expected standard deviation assuming pure white noise. For each light curve, we took $\beta$ to be the largest ratio among the bin sizes used and multiplied the individual error bars by that factor. We took $\beta$ to be 1.0 when the time-averaging method gave a smaller value. The values of $\alpha$ and $\beta$ for each light curve are listed in Table 2.

To determine the "best" values of the parameters and their uncertainties, we used a Monte Carlo Markov Chain (MCMC) algorithm (e.g., Tegmark et al. 2004; Ford 2005) with Metropolis-Hastings sampling, which has become the standard practice in the literature on transit photometry (Holman et al. 2006; Collier Cameron et al. 2007; Burke et al. 2007). We used here an adaptive approach, similar to the one described by Shporer et al. (2009). The algorithm steps from a multidimensional point in parameter space, $\bar{P}_{i}$, to the next, $\bar{P}_{i+1}$, according to

$$
\bar{P}_{i+1}=\bar{P}_{i}+f \bar{\sigma} \bar{G}(0,1),
$$

where $\bar{G}(0,1)$ is a vector of numbers picked randomly from a Gaussian distribution of zero mean and unit standard deviation, $\bar{\sigma}$ is a vector of the so-called step sizes, and $f$ (the only scalar in Equation (5)) is a factor chosen to control the fraction of accepted steps. The value of $f$ was readjusted every $10^{3}$ steps, to keep the acceptance fraction near 25\% (Gregory 2005). Our final MCMC included 10 long chains of 500,000 steps each, starting from different initial positions in parameter space spaced apart by $\approx 5 \sigma$ from the best-fitting parameters. The posterior probability distribution of each parameter was constructed from all long chains after discarding the first $20 \%$ of the steps. We took the distribution median to be the "best" value and the values at the 84.13 and 15.87 percentiles to be the $+1 \sigma$ and $-1 \sigma$ confidence uncertainties, respectively.

\section{RESULTS}

Results of the fitting process are presented visually in Figure 1 where each light curve is plotted separately and overplotted by the fitted model, using limb-darkening coefficients of the corresponding filter. Figure 2 shows the combined light curve, with the 2010 January 13 and Sep09 light curves plotted on top of each other.

The fitted model for the Jan 10 event has $\chi^{2} / N_{\text {dof }}=442 / 429$ and for the Sep09 event was $\chi^{2} / N_{\text {dof }}=36 / 33$. The rms residual of each binned light curve is given in the second-to-last column of Table 2. The rightmost column of Table 2 lists the photometric noise rate (PNR) of the unbinned light curve, defined as the rms divided by $\sqrt{\Gamma}$, where $\Gamma$ is the median number of data points per unit time (the cadence). The PNR is meant to be a quantitative comparison between the statistical power of different datasets (see also Burke et al. 2008 and Shporer et al. 2009 ${ }^{31}$ ).

The fitting process resulted in 10 estimates of the periastron passage time, $\left\{T_{p, i}\right\}_{i=1}^{10}$. Using the resulting distributions of the light-curve parameters, we numerically translated each periastron time to midtransit time. We then averaged the midtransit times to get our final estimate of the Jan10 midtransit time, $T_{c, \text { Jan10 }}$. While examining the ten individual midtransit time estimates, we noticed that three of them have relatively large uncertainties, larger than 0.015 days, while the rest have uncertainties in the range of $0.005-0.010$ days. Therefore, we removed them from the sample before averaging. Those three are the OHP, RHO, and FLWO light curves, spanning less than $5 \mathrm{hr}$ without any out-of-transit data; hence, the increased uncertainties are expected. We used an unweighted average to get our final midtransit time estimate, of $T_{c}$, Jan10 $[\mathrm{HJD}]=2455210.6502$, with an $\mathrm{rms}$ of 0.0064 days, close to the typical uncertainty of the seven individual estimates. Using regular averaging acts to average out possible correlated noise affecting individual estimates. Using median or weighted average changes the midtransit time by $\lesssim 0.25 \sigma$. Including the three large uncertainty $T_{c, i}$ 's mentioned above also results in a small change to $T_{c, \text { Jan10, }}$, of less than $0.3 \sigma$, but the scatter is increased by $50 \%$.

To check the sensitivity of our result to our treatment of correlated noise, we reran our analysis for the Jan10 data while using $\beta=1.0$ for all light curves. The resulting $T_{c, i}$ uncertainties were smaller by typically $25 \%-30 \%$; and the average midtransit time was $2455210.6476 \pm 0.0093$, earlier by $0.4 \sigma$ than our preferred analysis.

For estimating the midtransit time of the Sep09 event,

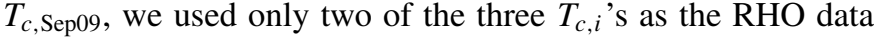
are entirely out-of-transit. The average between these two is $T_{c \text {,Sep09}}[\mathrm{HJD}]=2455099.196$, and we assign it an uncertainty of 0.026 days, similar to that of the two $T_{c, i}$ 's.

We note that Figures 1 and 2 were produced using the average midtransit times of each event, derived above, not with the $T_{c, i}$ of each partial light curve.

\section{DISCUSSION}

Table 3 lists the midtransit times derived here: the one measured by Hébrard et al. (2010) and those predicted by Winn et al. (2009). For the Jan10 event, Hébrard et al. (2010) measured a $T_{c, \operatorname{Jan} 10}$ which is $3 \sigma$ earlier than predicted by Winn et al. (2009). Our measured $T_{c \text {, Jan10 }}$ is intermediate between those of Hébrard et al. (2010) and Winn et al. (2009). It is $1.3 \sigma$ later than the Hébrard et al. (2010) time and earlier by $1.1 \sigma$ than the Winn et al. (2009) prediction. Therefore, it does not confirm nor refute the earlier transit time measured by Hébrard et al. (2010).

\footnotetext{
31 We note that the equation for the PNR given by Shporer et al. (2009) includes a typographical error, although their calculations are correct.
} 

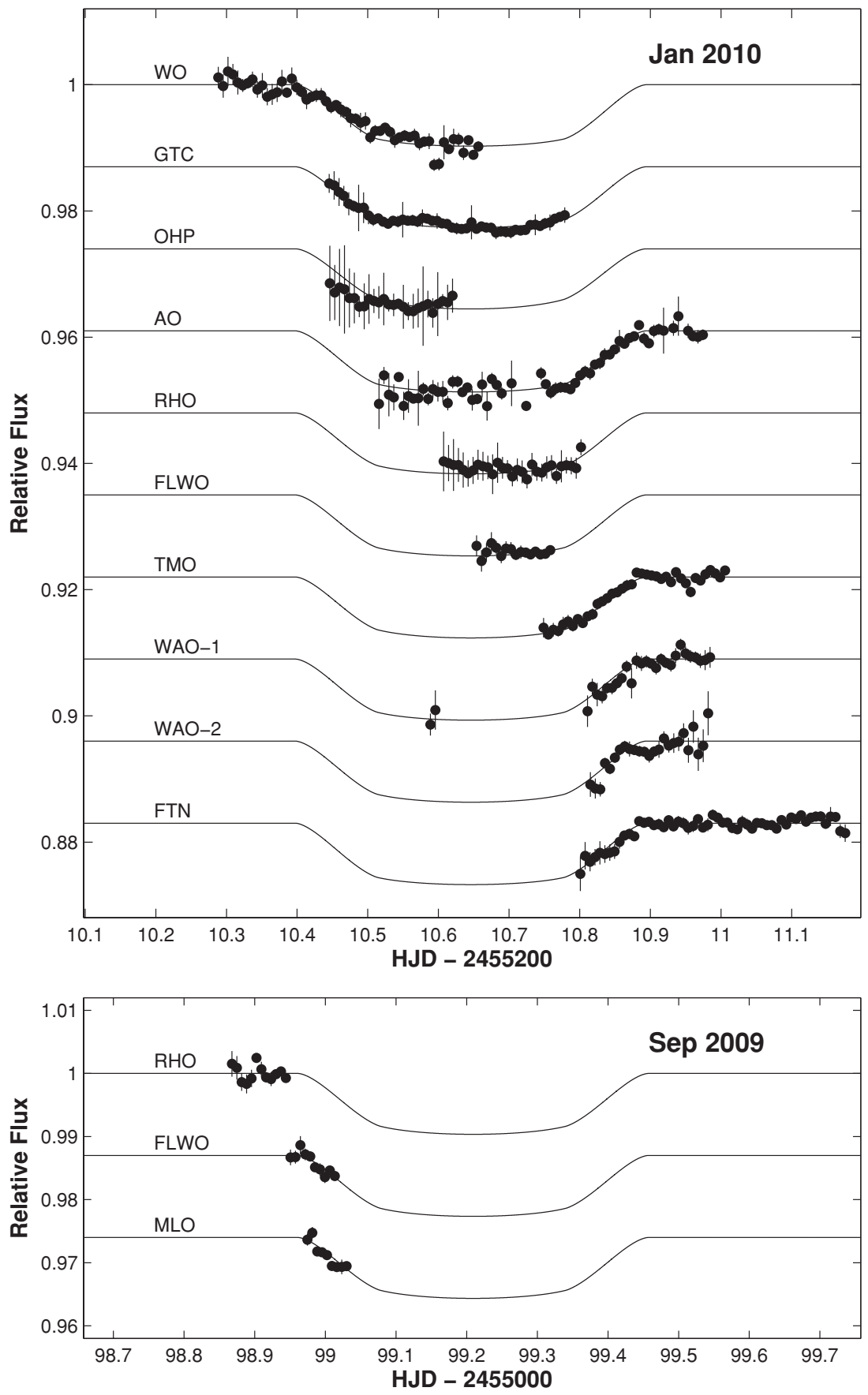

Figure 1. New light curves of HD 80606. Top: the ten light curves of the Jan10 event. Bottom: the three light curves of the Sep09 event. Each light curve is overplotted by the best-fitting model, with limb-darkening coefficients appropriate for the observing bandpass.

Table 3

Midtransit Times

\begin{tabular}{|c|c|}
\hline Reference & $T_{c}[\mathrm{HJD}]$ \\
\hline \multicolumn{2}{|c|}{$2010 \mathrm{Jan}$} \\
\hline This work & $2455210.6502(64)$ \\
\hline Hébrard et al. 2010 & $2455210.6420(10)$ \\
\hline Winn et al. $2009^{\mathrm{a}}$ & $2455210.6590(51)$ \\
\hline \multicolumn{2}{|c|}{$2009 \mathrm{Sep}$} \\
\hline This work & $2455099.196(26)$ \\
\hline Winn et al. $2009^{\mathrm{a}}$ & $2455099.2216(50)$ \\
\hline
\end{tabular}

Note. ${ }^{\text {a }}$ Predicted $T_{c}$ based on the ephemeris given in that reference.
There is some discrepancy between Hébrard et al. (2010) and Winn et al. (2009) also in the three parameters determining the light-curve shape, namely $r, a / R_{\mathrm{s}}$, and $i$. Specifically, the values for the planet-star radius ratio differ by about $2.5 \sigma$, and for $a / R_{\mathrm{s}}$ and $i$ the difference is at the $1.5 \sigma$ level. To check which set of values is preferred by our data, we reran our analysis using the Winn et al. (2009) values for these three parameters as prior constraints in Equation (3). This resulted in an increased $\chi^{2} / N_{\text {dof }}$ of $40 \%$, showing that our data prefer the Hébrard et al. (2010) values. This indicates that the discrepancy is not the result of a wavelength-dependent radius ratio and more likely results from underestimated uncertainties. 


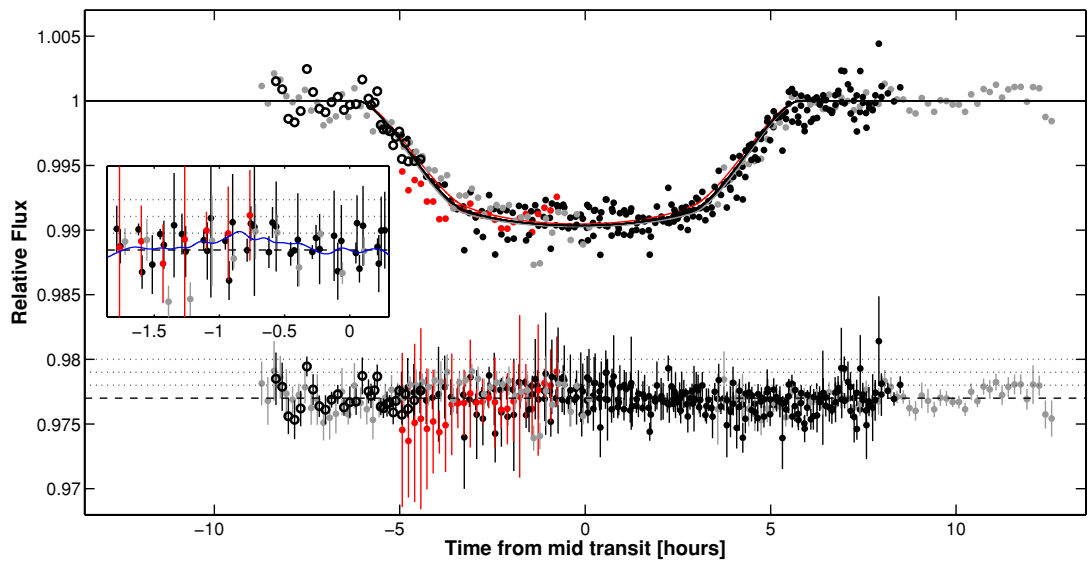

Figure 2. Composite light curve. The phase-folded light curve based on the 13 new datasets analyzed in this paper. Light curves taken with different filters are represented by different colors. For each filter, the corresponding model light curve is plotted as a solid line with the same color. Data obtained in the $Z$ band are in gray, $i$ and $I$ data in black, and $r$ data in red. Open circles represent the Sep09 data and filled circles the Jan10 data. Plotted below the light curve are the residuals, with error bars. The dashed line marks the residual zero point, and the three dotted lines mark relative flux residual levels of $0.1 \%, 0.2 \%$, and $0.3 \%$. The inset shows a zoomed-in view of the residuals during the phase when a "rebrightening" or "bump" was observed with Spitzer by Hébrard et al. (2010). The Spitzer feature is overplotted in blue. Our data neither confirm nor refute the existence of the bump.

(A color version of this figure is available in the online journal.)

Hébrard et al. (2010) noticed a "bump" in their Spitzer light curve: a $\sim 0.1 \%$ increase in flux lasting about $1 \mathrm{hr}$, just before midtransit. This bump could be caused by the passage of the planet in front of a dark spot on the star, although HD 80606 is not known to be an active star (Hébrard et al. 2010). The decreased surface brightness of a spotted surface element results from decreased temperature relative to a non-spotted surface element, so the flux increase during a spot crossing is expected to be wavelength dependent, increasing with shorter wavelengths (Pont et al. 2008; Rabus et al. 2009).

The inset in Figure 2 shows that our data are not sensitive enough to identify a $0.1 \%$ flux variation, although it does show that a $0.2 \%$ increase is unlikely. We note that another phenomenon that could, in principle, result in a small brief wavelength-independent bump is a triple conjunction of the star, planet, and a moon orbiting the planet (e.g., Sartoretti $\&$ Schneider 1999; Simon et al. 2010). A moon orbiting the planet could also be responsible for a shifted midtransit time (e.g., Sartoretti \& Schneider 1999; Simon et al. 2007). Although the existence of a moon is an exciting possibility, we caution that it is not likely to be the case in the current system due to the improbability of a triple conjunction, and perhaps also the dynamical instability of such a scenario.

We have presented here the results of an observational multisite campaign, along with a method to combine the partial ground-based light curves to obtain a complete transit light curve of a planet with a long-period and long-transit duration. Our results in comparison with the simultaneous Spitzer observations of Hébrard et al. (2010) allow an assessment of the quality of multisite campaigns and develop this method for the future discoveries of additional systems with similar characteristics. Such systems will be discovered by photometric observations of stars known to have planets from RV surveys, like HD 80606 itself, and by the spaced-based transiting planet hunters CoRoT and Kepler, capable of continuous photometric monitoring. The recent discovery of CoRoT-9b (Deeg et al. 2010), with a 95 day period and an $8 \mathrm{hr}$ long transit, is an excellent example. Only two transit events were observed by CoRoT, allowing accurate determination of the light-curve shape parameters, while ground-based photometric follow-up observations were needed to refine the transit ephemeris. Using accurate light-curve parameters, determined by space-based observations, to measure a midtransit time of a ground-based light curve is similar to the approach we have taken here.

Ground-based observations are limited by effects of the atmosphere and short observing windows (the latter leading to the need to combine data obtained by different instruments), but small-to-medium telescopes are relatively easily accessible, compared to space telescopes. Although forming a collaboration between several observatories is not a trivial task, those will be motivated by the increase in transit timing variations with orbital period (e.g., Holman \& Murray 2005; Agol et al. 2005), thus allowing ground-based observations to detect the effects of an additional, unseen planet in the system.

Some of the limitations and difficulties mentioned above can be minimized by using a network of identical instruments spread around the globe, allowing for continuous (24/7) monitoring of the target, especially when the observing windows partially overlap, allowing an accurate combination of the partial light curves. The LCOGT network (e.g., Brown et al. 2010; Lewis et al. 2010), once completed, is meant to be such a network, in both the Northern and Southern hemispheres. For targets in Northern (or Southern) positions such as HD 80606, the observational windows will partially overlap.

We thank John Caldwell, Steve Odewahn (McDonald Observatory, TX, USA), James Otto, Ohad Shemmer (Monroe Observatory, TX, USA), and Anthony Ayiomamitis (Hellenic Astronomical Union, Greece) for their attempt to observe HD 80606 during the Jan 10 event, although they were unable to do so due to bad weather. This paper uses observations obtained with facilities of the Las Cumbres Observatory Global Telescope. The MONET network is funded by the Alfried Krupp von Bohlen und Halbach-Stiftung. RHO observations were supported by the University of Florida and the College of Liberal Arts and Sciences. K.D.C. is supported by an NSF Graduate Research Fellowship. This material is based upon work supported by the National Science Foundation under grant No. 0707203.

This work is partially based on observations made with the Gran Telescopio Canarias (GTC), installed in the Spanish 
Observatorio del Roque de los Muchachos of the Instituto de Astrofísica de Canarias, on the island of La Palma. The GTC is a joint initiative of Spain (led by the Instituto de Astrofísica de Canarias), the University of Florida and Mexico, including the Instituto de Astronomía de la Universidad Nacional Autónoma de México (IA-UNAM) and Instituto Nacional de Astrofísica, Óptica y Electrónica (INAOE). K.D.C., H.J.D., and E.B.F. gratefully acknowledge the observing staff at the GTC and give a special thanks to René Rutten, José Miguel González, Jordi Cepa Nogué, and Daniel Reverte for helping us plan and conduct the GTC observations successfully. H.J.D. acknowledges support by grant ESP2007-65480-C02-02 of the Spanish Ministerio de Ciencia e Inovación. J.N.W. gratefully acknowledges support from the NASA Origins program through award NNX09AB33G and from the MIT Class of 1942. This research was partly supported by the Israel Science Foundation (grant No. 655/07) and by the United States-Israel Binational Science Foundation (BSF) grant No. 2006234.

Facilities: FTN (Spectral), GTC (OSIRIS)

\section{REFERENCES}

Agol, E., Steffen, J., Sari, R., \& Clarkson, W. 2005, MNRAS, 359, 567

Brown, T. M., et al. 2010, BAAS, 41, 401

Burke, C. J., et al. 2007, ApJ, 671, 2115

Burke, C. J., et al. 2008, ApJ, 686, 1331

Carter, J. A., \& Winn, J. N. 2009, ApJ, 704, 51

Claret, A. 2000, A\&A, 363, 1081

Claret, A. 2004, A\&A, 428, 1001

Collier Cameron, A., et al. 2007, MNRAS, 380, 1230

Colón, K. D., Ford, E. B., Redfield, S., Fortney, J. J., Shabram, M., Deeg, H. J., \& Mahadevan, S. 2010, MNRAS, submitted (arXiv:1008.4800)
Deeg, H. J., et al. 2010, Nature, 464, 384

Eastman, J., Siverd, R., \& Gaudi, B. S. 2010, PASP, 122, 935

Fabrycky, D., \& Tremaine, S. 2007, ApJ, 669, 1298

Ford, E. B. 2005, AJ, 129, 1706

Fossey, S. J., Waldmann, I. P., \& Kipping, D. M. 2009, MNRAS, 396, L16

Garcia-Melendo, E., \& McCullough, P. R. 2009, ApJ, 698, 558

Gregory, P. C. 2005, ApJ, 631, 1198

Hébrard, G., et al. 2010, A\&A, 516, A95

Hidas, M. G., et al. 2010, MNRAS, 406, 1146

Holman, M. J., \& Murray, N. W. 2005, Science, 307, 1288

Holman, M. J., et al. 2006, ApJ, 652, 1715

Knutson, H. A., et al. 2009, ApJ, 690, 822

Laughlin, G., Deming, D., Langton, J., Kasen, D., Vogt, S., Butler, P., Rivera, E., \& Meschiari, S. 2009, Nature, 457, 562

Lewis, F., Street, R., Roche, P., Stroud, V., \& Russell, D. M. 2010, Adv. Astron., 2010

Mandel, K., \& Agol, E. 2002, ApJ, 580, L171

Moutou, C., et al. 2009, A\&A, 498, L5

Naef, D., et al. 2001, A\&A, 375, L27

Nesvorný, D., \& Beaugé, C. 2010, ApJ, 709, L44

Pont, F., Knutson, H., Gilliland, R. L., Moutou, C., \& Charbonneau, D. 2008, MNRAS, 385, 109

Pont, F., Zucker, S., \& Queloz, D. 2006, MNRAS, 373, 231

Pont, F., et al. 2009, A\&A, 502, 695

Rabus, M., et al. 2009, A\&A, 494, 391

Sartoretti, P., \& Schneider, J. 1999, A\&AS, 134, 553

Shporer, A., Mazeh, T., Pont, F., Winn, J. N., Holman, M. J., Latham, D. W., \& Esquerdo, G. A. 2009, ApJ, 694, 1559

Simon, A. E., Szabó, G. M., Szatmáry, K., \& Kiss, L. L. 2010, MNRAS, 406, 2038

Simon, A., Szatmáry, K., \& Szabó, G. M. 2007, A\&A, 470, 727

Sybilski, P., Konacki, M., \& Kozłowski, S. 2010, MNRAS, 405, 657

Tegmark, M., et al. 2004, Phys. Rev. D, 69, 103501

Triaud, A. H. M. J., et al. 2010, A\&A, in press (arXiv:1008.2353)

Winn, J. N., et al. 2008, ApJ, 683, 1076

Winn, J. N., et al. 2009, ApJ, 703, 2091

Wu, Y., \& Murray, N. 2003, ApJ, 589, 605 